\title{
ELECTRICAL CONDUCTIVITY FOR THE STRONGLY CORRELATED AND HYBRIDIZED HUBBARD BAND
}

\author{
R. Kosimow ${ }^{a}$, W. Borgies ${ }^{a}$ AND J. DeniszCZYK ${ }^{b}$ \\ ${ }^{a}$ Institute of Physics, Silesian University \\ Universytecka 4, 40-007 Katowice, Poland \\ ${ }^{b}$ Institute of Physics and Chemistry of Metals, Silesian University \\ Bankowa 12, 40-007 Katowice, Poland
}

The magnetic and electrical transport properties of strongly correlated Hubbard band ( $f$-band) hybridizing with the non-correlated conduction band (c-band) was studied. The many-body model Hamiltonian was treated within the modified alloy analogy scheme and the resulting one-body problem was solved with the use of coherent potential approximation. Within the Kubo-Greenwood formalism the dc electrical conductivity for bcc magnetic system was calculated and its variation with temperature and number of electrons was examined for different values of the Coulomb and hybridization $(V)$ coupling constants. We found that the effect of strong correlations and hybridization on the conductivity is similar to that produced by the scattering due to the $s-f$ interaction (spin disorder resistivity).

PACS numbers: 71.10.Fd, 71.27.+a, 72.10.-d, 72.15.-v

\section{Introduction}

Generally, it is a difficult task to calculate direct influence of the Coulomb correlations on the transport properties especially on electrical conductivity [1]. In the present paper we analyze the problem within the Hubbard model in which $f$-electrons additionally hybridize with separated non-magnetic $c$-band. The strong Coulomb correlations can force the magnetic order in the system and the electrons responsible for electrical transport will undergo the additional scattering on the effective magnetic moments. Our aim is to study the influence of $c$-electron band on the magnetic and transport properties of the system.

\section{Calculations and results}

Let us consider the two-band model described by the Hamiltonian

$$
H=H_{c}+H_{f}+H_{c f}
$$

where

$$
H_{c}=\sum_{i, j, \sigma} T_{i j}^{c} c_{i \sigma}^{+} c_{j \sigma}
$$




$$
\begin{aligned}
& H_{f}=\sum_{i, j, \sigma}\left(T_{i j}^{f}-\mu\right) f_{i \sigma}^{+} f_{j \sigma}+\frac{1}{2} U \sum_{i \sigma} n_{i \sigma} n_{i-\sigma}, \\
& H_{c f}=V \sum_{i, \sigma}\left(f_{i \sigma}^{+} c_{i \sigma}+c_{i \sigma}^{+} f_{i \sigma}\right) .
\end{aligned}
$$

Hamiltonians (2) and (3) describe the $c$ and $f$ electron subsystems, respectively ( $\mu$ denotes the chemical potential and $U$ - the Coulomb repulsion). Hamiltonian (4) describes the hybridization between the two electronic subsystems. In the analytical evaluations we assumed the same dispersion relations for both subsystems but the different band widths were allowed (different values of hopping integrals $\left.T_{i j}^{c, f}\right)$.

We have treated the Hubbard Hamiltonian (3) for the $f$-electron within the modified alloy analogy (MAA) approach [2] which maps the Hamiltonian $H_{f}$ onto effective, single-particle one. As a result, Hamiltonian (1) was reduced to the one describing two single-particle subsystems coupled by hybridization interaction (4). This allowed us to solve the resulting set of the coupled Green function equations analytically without any further approximations. We have solved numerically the set of non-linear integral equations in the self-consistent way and we have got the self-energy function for the $f$-electrons modified by hybridization with $c$-electron states. Having it we were able to examine the magnetic and transport properties for the $f$-subsystem.

Generally, to develop the transport properties we need to know the two-particle Green functions which enter the Kubo formula for conductivity [3]. Within the CPA method for the case of one band system the two-particle Green function factorizes into the product of the one-particle ones [4] and the formula for the dc conductivity takes a simple form $[5,6]$. In the real systems the electrons from different bands contribute to the electric current operator and the problem of the vertex corrections even in the framework of CPA is not an easy task to handle [7]. In our approach we assumed a small hybridization between the two electronic subsystems and therefore we have neglected vertex corrections [7].

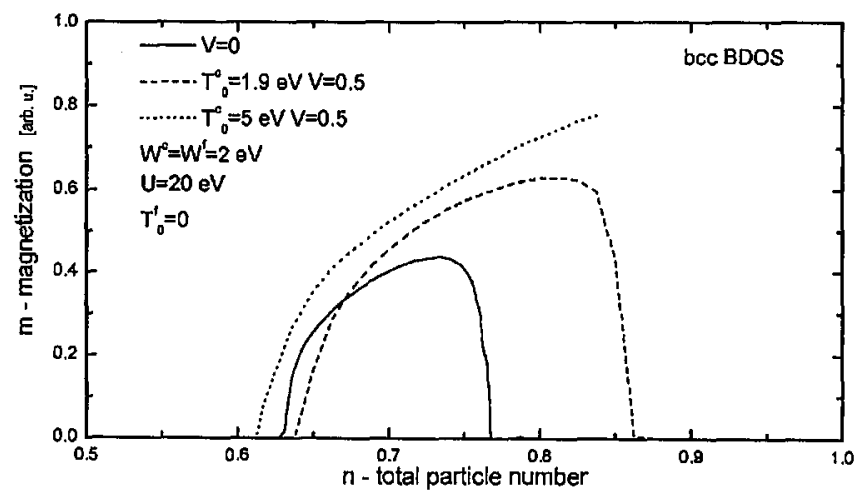

Fig. 1. Magnetization of the $f$-subsystem $-m$, as a function of the band occupation $n$, for different values of the centre of gravity $T_{0}^{c}$ of the $c$-band. 


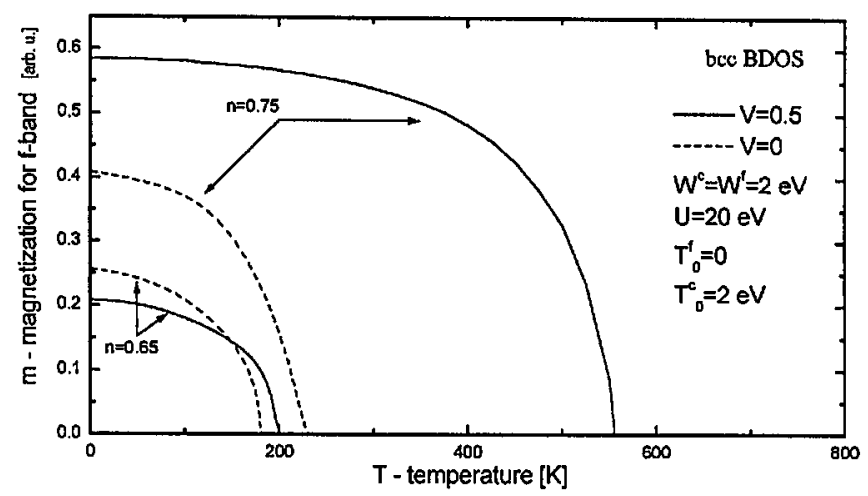

Fig. 2. Magnetization, as function of temperature $T$, for various values of the band occupation number $-n$.

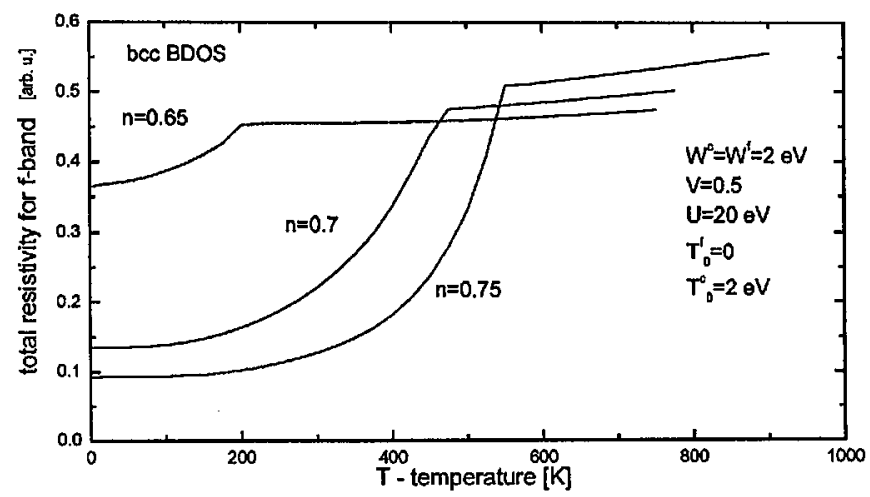

Fig. 3. Total $f$ electrons resistivity (inverse conductivity) as function of temperature for bcc BDOS lattice with different values of particle number.

We have performed numerical calculations for bcc lattice and different temperatures, band occupations numbers and magnetic phases of the system. The bcc Bloch density of state (DOS) function which was used as an input in the MAA procedure was taken from the interpolation recipe given by Jelitto [8]. The influence of hybridization $V$ for the electrons with spin $\sigma=\uparrow$ is much stronger than for $\sigma=\downarrow$ electrons. It is mainly because of much higher number of the electrons in the up spin subband. Therefore for the all considered cases the magnetization for system with hybridization was higher. Also the range of the particle number in the system for which ferromagnetic phase exists was significantly broadened (see Fig. 1).

Figure 1 shows that hybridization enhances drastically magnetization of the $f$ electrons and in some cases can even lead to the magnetic saturation in the $\sigma=\uparrow$ spin band. Magnetization also increases with increasing distances between the centers of the gravity for the $c$ and $f$ bands. It grows up to the limiting values of band separation. The magnetization as function of the temperature is plotted in Fig. 2. The values of the Curie temperature $T_{\mathrm{C}}$ are all below $1000 \mathrm{~K}$ and are 
quite realistic when compared with experiments. The values of magnetization with vanishing hybridization are also included (dashed lines in Fig. 2). The temperature dependence of resistivity is plotted in Fig. 3 .

\section{Conclusions}

The possibility of a spontaneous magnetic order, and dc electrical conductivity for strongly correlated Hubbard's band which hybridizes with conducting band was investigated within MAA. We have found that the additional band has an important influence on both fundamental properties. In general, the hybridization in the mutual configurations of the bands we have analyzed always increases $f$-band magnetization and the Curie temperature $T_{\mathrm{C}}$. The system with hybridization was more stable and convergence of numerical calculations was quicker.

The temperature dependence of resistivity (Fig. 3 ) is qualitatively very similar to those of metallic rare-earth elements and their alloys [9]. The resistivity at low temperatures $\left(0-0.75 T_{\mathrm{C}}\right)$ exhibits a very accurate power-law $\left(\sim T^{3}\right)$ behaviour and for temperatures close to $T_{\mathrm{C}}$ shows the characteristic critical behaviour. This type of low temperature behaviour of resistivity is observed experimentally in rare-earth alloys [10]. The finite values of the conductivity at $T=0 \mathrm{~K}$ results from the virtual spin flip scattering and non-zero hybridization. We have to admit that residual resistivity vanishes when saturation of the $f$ magnetization is reached. Additional investigations which will clarify unusual behaviour of the residual resistivity at $T=0 \mathrm{~K}$ are necessary. The improved model with the more accurate self-energy is under consideration.

\section{Acknowledgments}

This work was supported in part by the Committee for Scientific Research under contract No. 2 P03B 12914.

\section{References}

[1] M. Kaveh, N. Wiser, Adv. Phys. 33, 257 (1984).

[2] T. Herrmann, W. Nolting, Phys. Rev. B 53, 10579 (1996).

[3] R. Kubo, J. Phys. Soc. Japan 12, 570 (1957).

[4] B. Velický, Phys. Rev. 184, 614 (1969).

[5] V.I. Grebennikov, Y.I. Prokopjev, Sov. Phys.-Phys. Metal. Metalloved 60, 213 (1985).

[6] T. Herrmann, R. Kosimow, W. Borgieł, W. Nolting, in preparation.

[7] P.N. Sen, Phys. Rev. B 8, 5613 (1973).

[8] R.J. Jelitto, J. Phys. Chem. Solids 30, 609 (1969).

[9] T. van Peski-Tinbergen, A.J. Dekker, Physica 29, 917 (1963).

[10] E. Talik, Physica B 193, 213 (1994). 